\title{
Interfacial Passivation of Perovskite Solar Cells by Reactive Ion Scavengers
}

\author{
Hakimeh Teymourinia, ${ }^{\dagger}$ Cedric Gonzales, ${ }^{\dagger}$ Juan Jesús Gallardo, Masoud Salavati-Niasari,* Juan Bisquert, \\ Javier Navas, and Antonio Guerrero*
}

Cite This: ACS Appl. Energy Mater. 2021, 4, 1078-1084

Read Online

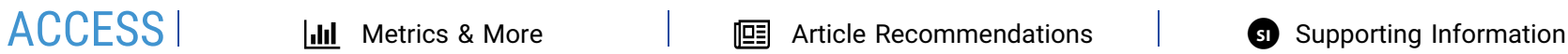

ABSTRACT: Lead halide perovskites suffer from uncontrolled ion migration and the interactions at the external contacts play a fundamental role in the hysteretic response and performance degradation kinetics. In this work, we passivate the external interfaces by a reaction of migrating iodide ions with a silver buffer layer placed between Spiro-OMeTAD and Au layers. In the presence of an electrical field, iodine migration occurs and ions that are close to the perovskite/contact interface irreversibly form a layer of AgI. Overall, the interfacial reaction of iodide ions totally suppresses hysteresis and leads to more stable devices. A new sample preparation method unburies the reactive interface, which is then probed by $\mathrm{X}$-ray photoelectron spectroscopy measurements. The kinetics of the layer formation are monitored by

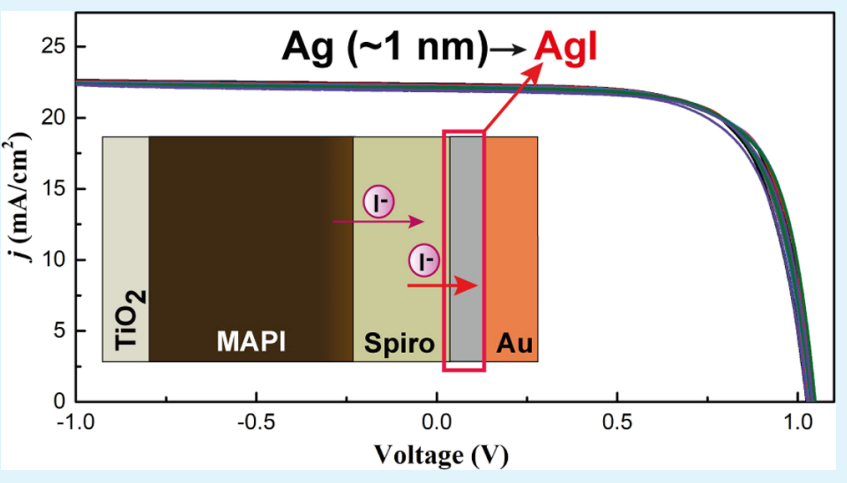
impedance spectroscopy highlighting that in the presence of an electrical field and light, the reaction occurs in the order of minutes. We further identify the resistive response of AgI in operating devices. The present work provides a new approach to passivate the external interfaces in lead halide perovskites.

KEYWORDS: perovskite, passivation, hysteresis, silver iodide, interfacial reactivity, impedance spectroscopy

\section{INTRODUCTION}

Hybrid organic-inorganic perovskite materials have gained enormous attention due to their exceptional optoelectronic properties for applications in solar cells, light emission diodes, $\mathrm{X}$-ray detectors, memory devices, etc. The chemical formula of perovskite can be represented as $\mathrm{ABX}_{3}$ where $\mathrm{A}=$ monovalent cations (i.e., methylammonium (MA)), $\mathrm{B}=$ divalent cations (typically $\mathrm{Pb}$ ), and $\mathrm{X}=$ halide anions $\left(\mathrm{Cl}^{-}, \mathrm{Br}^{-}\right.$, and $\left.\mathrm{I}^{-}\right)$. In the photovoltaic configuration, certified power conversion efficiencies (PCEs) of above $25 \%$ have been reached in less than 10 years by engineering the perovskite formulation and by improving the charge extraction at the contacts. ${ }^{1}$ Despite these improvements in efficiencies, unacceptable performance degradation rates still remain as one of the main challenges for commercial applications.

The well-understood degradation pathways include those involving the reactivity of the perovskite layer with water/ oxygen and those connected to photodegradation and phase segregation in mixed cation/anion formulations. ${ }^{2-4}$ Alternatively, understanding the chemical effects induced by ion migration at the interfacial level and their connection with performance decay has proved to be very challenging. ${ }^{5-8}$ In particular, ions that migrate into the bulk of the perovskite layer may reach the external contacts leading to an irreversible reaction with top metals (i.e., $\mathrm{Ca}, \mathrm{Al}$, or $\mathrm{Ag})^{9-12}$ and oxides (i.e., $\mathrm{SnO}_{2}$ or $\left.\mathrm{NiO}\right) .^{13}$ For example, layers of $\mathrm{Ag}(100 \mathrm{~nm})$ have been used as charge collectors by forming AgI due to the migration of ions during device operation. ${ }^{14,15}$ This newly formed layer is an electrically insulating material that blocks charge extraction and significantly reduces the device performance. Similarly, the reversible reaction with other materials such as $\mathrm{Au}$ or $\mathrm{TiO}_{2}$ has also been reported and is connected to hysteresis in the electrical response and degradation. ${ }^{16-21}$

Formation of these new species is directional as the source of ions is in the bulk of the perovskite layer and it has been difficult to develop techniques to reliably measure the formation of products at the interfacial level. For example, Xray photoelectron spectroscopy (XPS) is a surface-sensitive technique that would be well suited to analyze the elements present at interfaces together with their oxidation states and has been used in perovskite research. ${ }^{22}$ However, the interfaces are not accessible as they are buried in the device. Similarly, detecting the electrical response of specific interfaces can only

Received: July 28, 2020

Accepted: January 25, 2021

Published: February 7, 2021 
a)

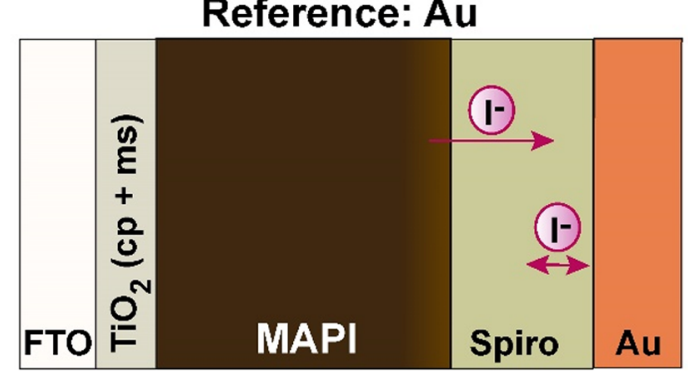

b)

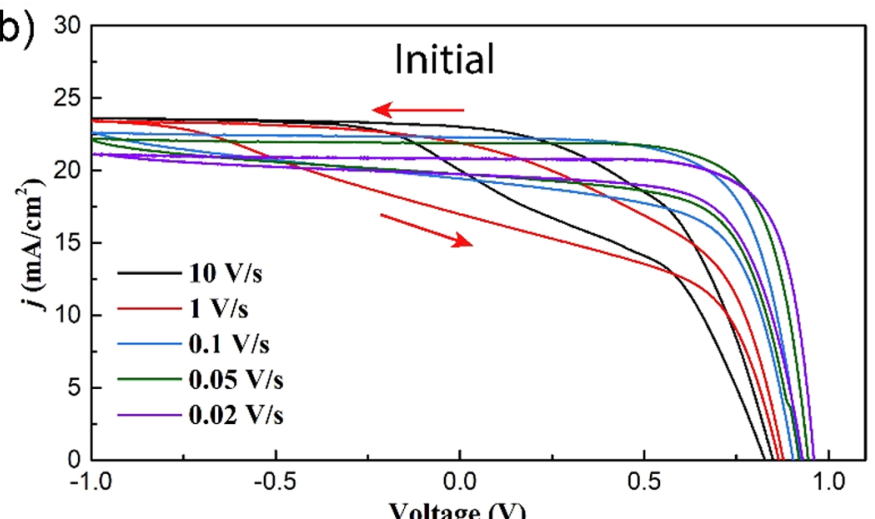

c)

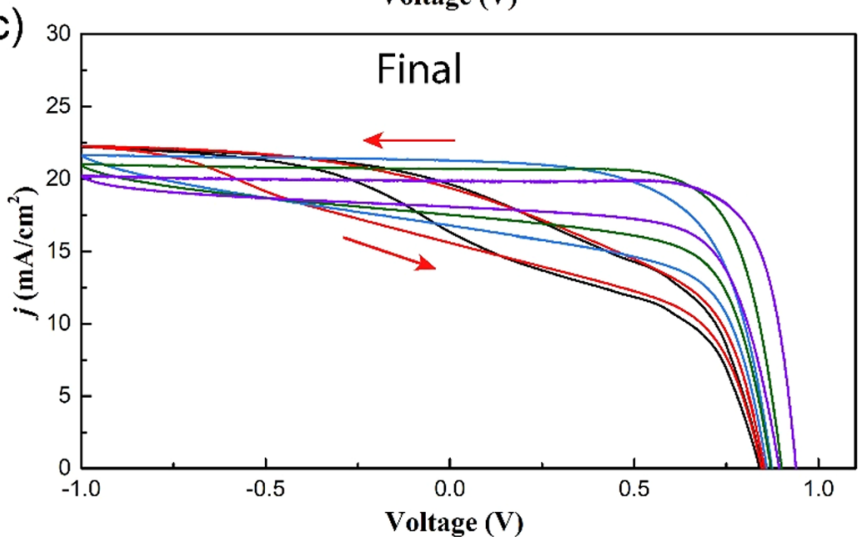

d)
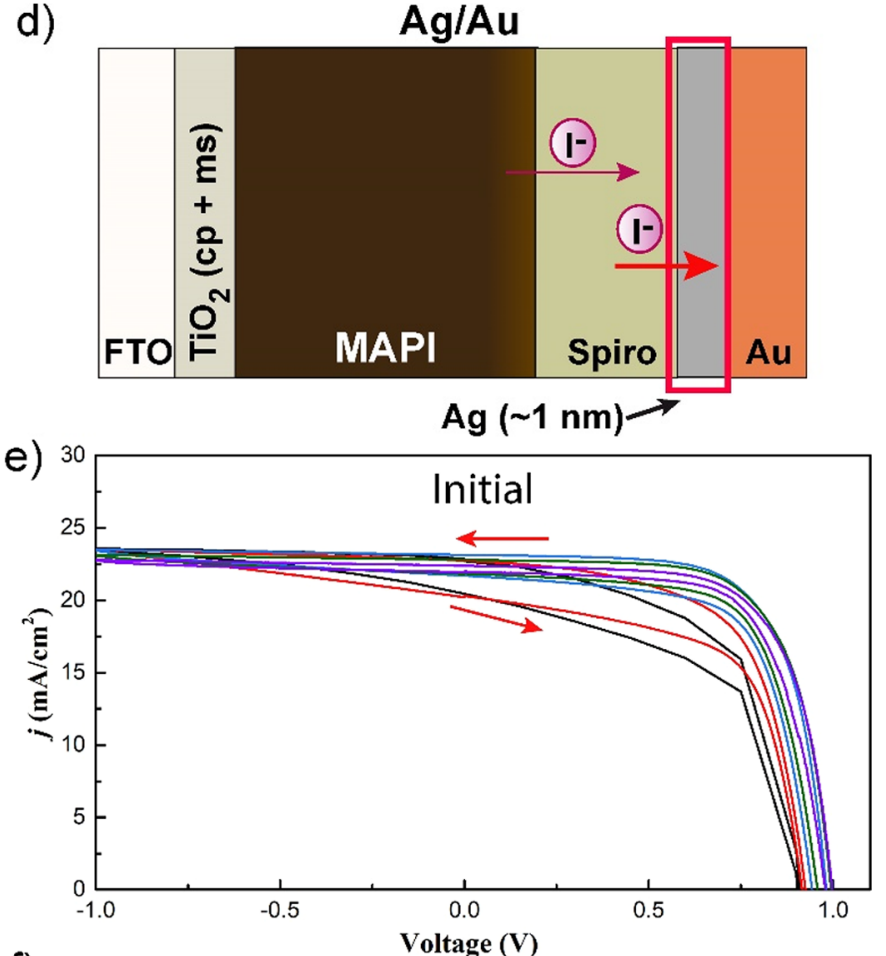

f)

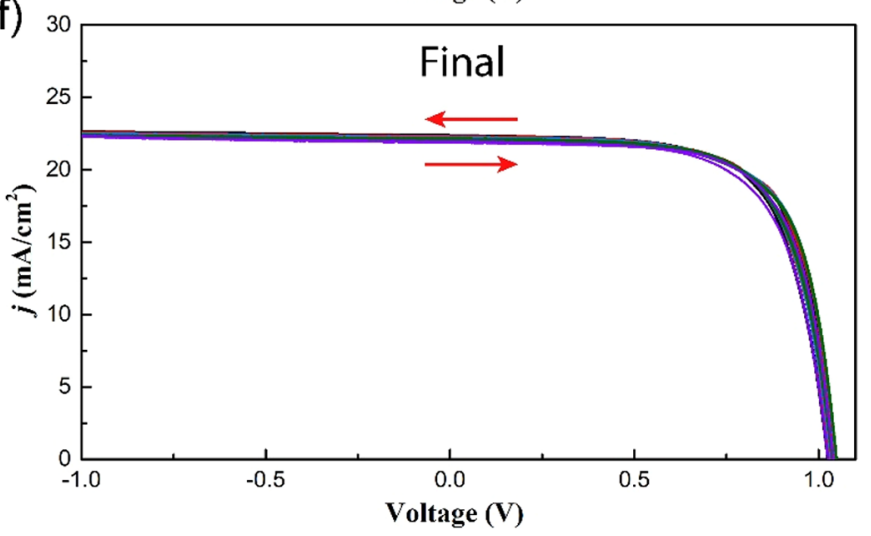

Figure 1. (a, d) Device configuration tested in this work. Representative $J-V$ curves of devices measured in a dry box at several scan rates (10 to 20 $\mathrm{mV} / \mathrm{s})$ at 1 sun illumination conditions, $(\mathrm{b}, \mathrm{e})$ fresh devices and $(\mathrm{c}, \mathrm{f})$ response after characterization using conditions that promote ion migration (illumination and voltage bias).

be probed by indirect techniques. These indirect measurements, supported by other analytical techniques, require electrical models to extract relevant information. ${ }^{9}$ Of particular interest is impedance spectroscopy (IS), an in operando technique that offers pertinent information on the kinetics of iodide ions: ion transport in the perovskite layer, ion accumulation at contacts, and chemical reactivity with contact materials. $^{23-26}$

In this work, we promote the reaction of migrating ions arising from the perovskite layer leading to hysteresis-free and more stable lead halide perovskite devices under illumination conditions. A silver buffer layer is placed between SpiroOMeTAD and the Au top contact with the aim of immobilizing iodide ions in the form of AgI. In this chemical reaction, $\operatorname{Ag}(0)$ is oxidized to $\operatorname{Ag}(\mathrm{I})$ and a complementary reduction reaction occurs within the device stack, which is not studied at this stage. Moreover, we develop a sample preparation method that provides access to the Spiro/top metal interface, which is then probed by XPS confirming the generation of AgI. Alternatively, by using IS, we are able to identify the electrical response of this individual interface in terms of the internal resistances associated with the AgI layer. Overall, we remove the hysteresis response due to the external interfaces in perovskite solar cells and monitor the formation of the AgI layer using electrical and analytical techniques that provide information at the interfacial level.

\section{RESULTS AND DISCUSSION}

Device Configuration and Electrical Testing. Figure 1 shows the two configurations studied in this work (Glass $\backslash$ FTO $\backslash \mathrm{TiO}_{2} \backslash \mathrm{MAPbI}_{3} \backslash$ Spiro-OMeTAD $\backslash$ metal contact). They differ in the metal contact, with one configuration having a thin layer of Ag placed between Spiro-OMeTAD and Au. The PCEs of the reference devices approach $17 \%$ in line with our previously reported results, and the statistics of 20 devices are provided in the Supporting Information. Similarly, a silver buffer layer with a thickness of $\sim 1 \mathrm{~nm}$ exhibits a comparable PCE. The main difference between the devices lies in the response toward 
a)

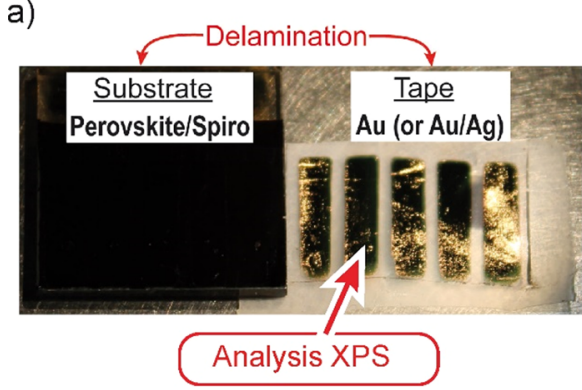

c)

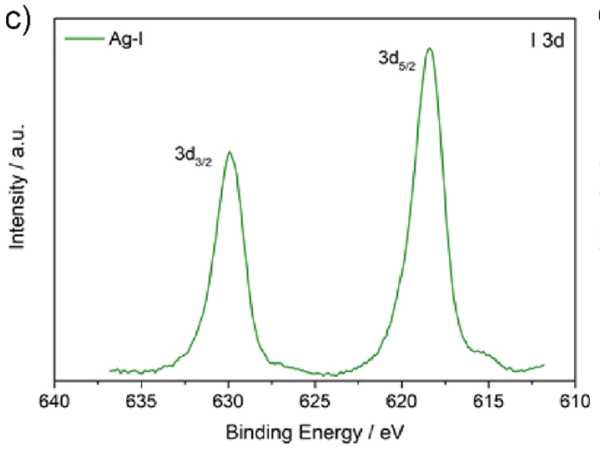

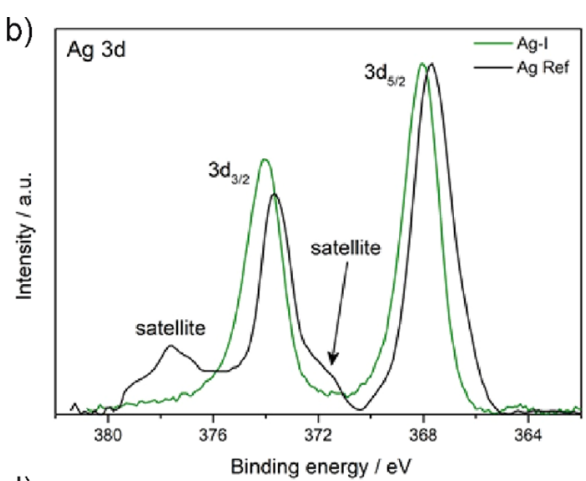

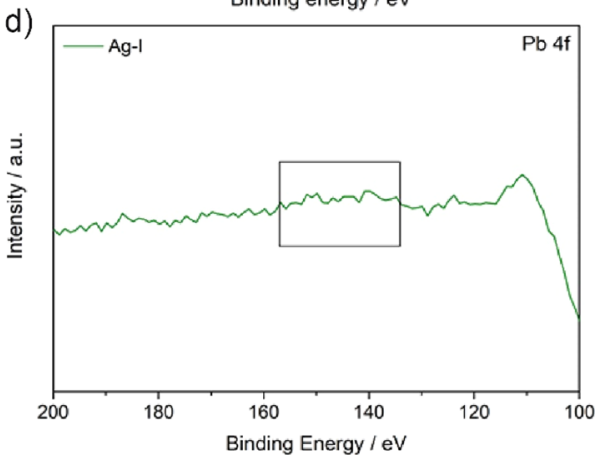

Figure 2. (a) Tape/contact delaminated samples prepared to study the XPS response. (b-d) XPS signals of the delaminated top contact in (a) obtained from a polarized photovoltaic device containing a buffer layer of $\mathrm{Ag}(2 \mathrm{~nm})$; the $\mathrm{Ag}$ signal is compared with a reference Ag metal sample.

hysteresis in the $J-V$ curves. The presence of hysteresis in the $J-V$ response is closely connected with the dynamic interaction of migrating ions with the external contacts, which not only modify the charge extraction properties but also lead to chemical reactions with the contacts. ${ }^{5,27}$ For example, we have recently reported the reversible reaction of migrating iodide ions with Au. ${ }^{16}$ The devices are characterized electrically following measurement protocols that polarize the fresh device at different scan rates under dark and light conditions, as detailed in the Supporting Information. In a typical experiment, $J-V$ curves are measured in the dark followed by light conditions (initial state), and the sequence is repeated once again to obtain $J-V$ curves in the final state. Here, both configurations initially show typical large hysteresis (Figure 1b,e) often reported in the literature. ${ }^{28,29}$ As reported previously, multiple measurement cycles induce the migration of iodide ions toward the top metal electrodes. ${ }^{25}$ Interestingly, while the reference device exhibits hysteresis (Figure 1c), devices containing a buffer layer of Ag show a large reduction of hysteresis (Figure 1f). Both polarization and light are required in the measurement protocol for the hysteresis reduction to take place in a time scale of $30 \mathrm{~min}$. Indeed, the same polarization protocol carried out in the dark does not lead to a reduction of hysteresis in that time scale. Interestingly, the reduction of hysteresis is not related to photogeneration and trap-related processes as the same reduction is observed for $J-V$ curves measured under dark conditions (see Supporting Information). Nevertheless, we note here that devices containing a Ag buffer layer stored in the dark for several days also show reduced hysteresis with time. Therefore, formation of AgI as described below is kinetically favored in the presence of light and an externally applied electrical field. It is important to note that devices containing a buffer layer of $\mathrm{Ag}$ show different dynamics for degradation compared to reference devices with increased stability
(Supporting Information). However, as expected for devices containing $\mathrm{MAPbI}_{3}$, degradation is very severe under 1 sun illumination and maximum power point tracking conditions in both cases.

Further information can be extracted from the $J-V$ curve analysis of devices fabricated with different thickness of $\mathrm{Ag}(\mathrm{Ag}$ $=0.5,2$, and $5 \mathrm{~nm}$ ), see Supporting Information. The beneficial effect of $\mathrm{Ag}$ on the suppression of $J-V$ hysteresis is not observed using a silver thickness of $0.5 \mathrm{~nm}$. On the other hand, a Ag thickness of $2 \mathrm{~nm}$ shows similar results to those observed for $1 \mathrm{~nm}$. Finally, devices containing a thick layer of $\mathrm{Ag}(5 \mathrm{~nm})$ not only does not suppress hysteresis but also leads to degradation of all performance parameters with a remarkable reduction in the photocurrent by about $4 \mathrm{~mA}$ / $\mathrm{cm}^{2}$ in the final state. Therefore, the silver thickness needs to be thick enough to capture all mobile ions but not too thick to prevent further induced degradation of the perovskite as discussed below.

We next set to understand the chemical modifications taking place at the interfacial level. The final state can be analyzed in terms of interfacial reactivity using a delamination method to clearly expose the Spiro-OMeTAD/top contact interface from photovoltaic devices. The delamination method has been developed in which the device (fresh or stressed) is covered using tape (3M) by pressing the adhesive side of the tape onto the top electrodes. The tape is then carefully removed and delamination is promoted at the weakest interface available, the Spiro-OMeTAD/top contact. This methodology leaves, on the one hand, a substrate with the perovskite and Spiro layer, and on the other hand, the tape with the top contact ( $\mathrm{Au}$ or $\mathrm{Ag}$ / $\mathrm{Au}$ ). The exposed surface of the metal corresponds to layers that were in contact with Spiro-OMeTAD, enabling the study of interfacial reactivity by the surface-sensitive technique XPS. Figure $2 \mathrm{~b}$ shows the Ag $3 \mathrm{~d}$ high-resolution XPS spectrum of the Ag sample that was stressed under bias and light compared 

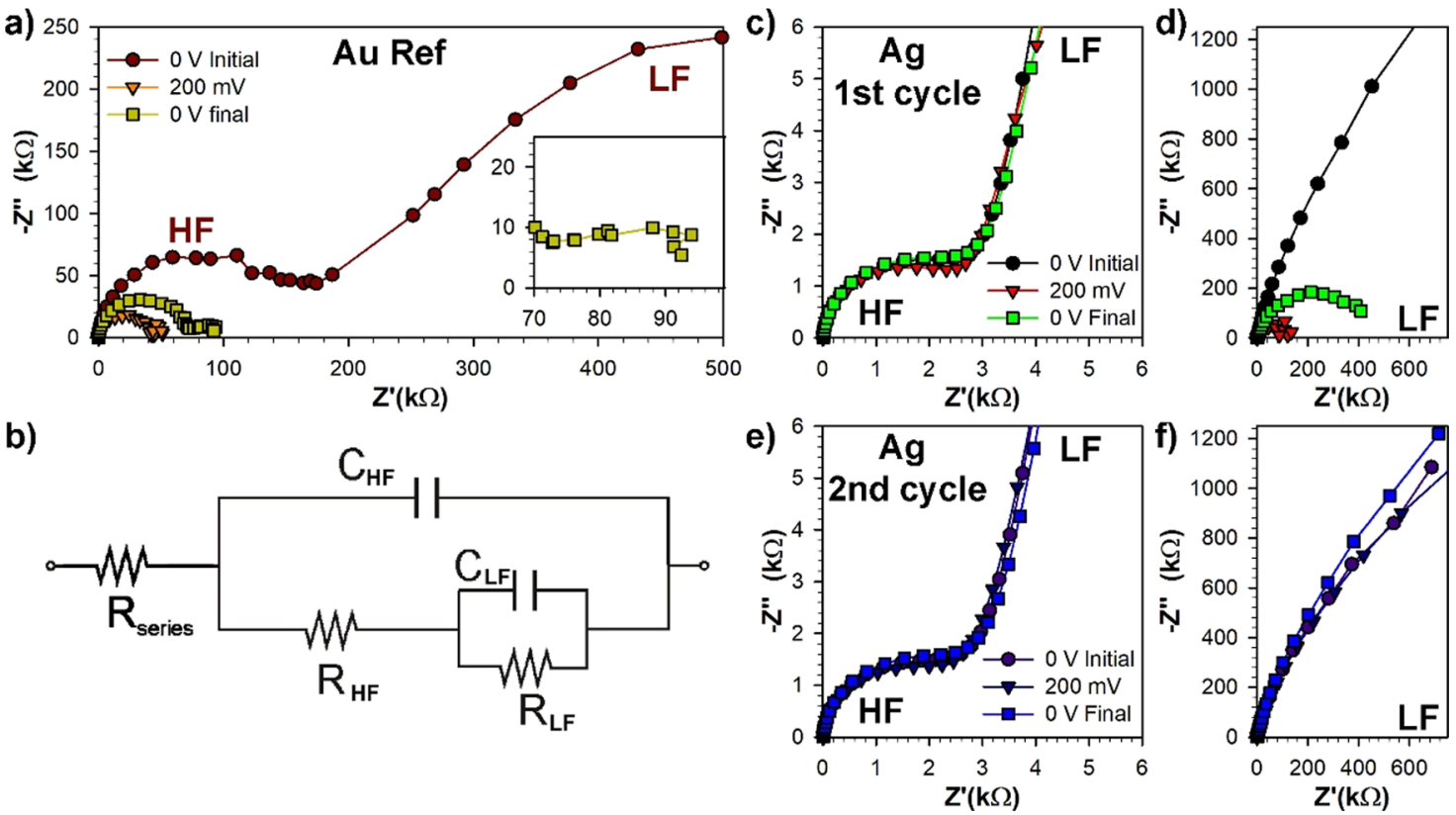

Figure 3. (a) IS spectra of a fresh reference device measured in the dark following the sequence $0 \mathrm{~V} \rightarrow 200 \mathrm{mV} \rightarrow 0 \mathrm{~V}$ and (b) equivalent circuit used to fit the data. (c, d) IS spectra of a device containing Ag as a buffer layer using the same protocol as in (a). The voltage sequence was repeated a second time for the same device and the results are shown in (e, f).

with a reference sample of $\mathrm{Ag}(100 \mathrm{~nm})$ on FTO. The spectrum of the $\mathrm{Ag}$ reference sample shows two well-defined peaks due to the $\mathrm{Ag} 3 \mathrm{~d}_{5 / 2}(367.9 \mathrm{eV})$ and $\mathrm{Ag} 3 \mathrm{~d}_{3 / 2}(373.9 \mathrm{eV})$ signals, in good agreement with the reported values for pure

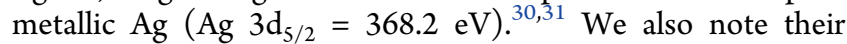
corresponding satellites signals at higher binding energies (BEs). ${ }^{31}$ A detailed discussion is provided in the Supporting Information. On the other hand, the device that has been electrically characterized (AgI) shows that the $\mathrm{Ag} 3 \mathrm{~d}$ signals are shifted to a higher $\mathrm{BE}$, which is evident of the presence of oxidized Ag species with values matching those reported for AgI. ${ }^{32-35}$ However, we highlight that given sufficient time and stress factors (light and externally applied voltage), all $\mathrm{Ag}$ would turn into AgI. Moreover, the I $3 \mathrm{~d}$ signal (Figure 2c) shows two peaks due to I $3 \mathrm{~d}_{5 / 2}(618.5 \mathrm{eV})$ and $\mathrm{I} 3 \mathrm{~d}_{3 / 2}(630.0$ $\mathrm{eV}$ ) signals. These $\mathrm{BE}$ values are typical for the presence of $\mathrm{I}^{-}$ species and prove the diffusion of iodide ions across the SpiroOMeTAD layer leading to the formation of $\mathrm{Ag}-\mathrm{I}$ bonds. ${ }^{30,35}$ Additionally, the separation between both spin-orbit components for the I $3 \mathrm{~d}$ signal matches exactly to the reported values of $11.5 \mathrm{eV}$ for AgI. ${ }^{37}$ Also, as the amount of iodide and the shape of the Ag peaks are very similar for both samples analyzed (see Figure S6 in the Supporting Information), very little metallic silver is expected to remain in the film. ${ }^{22}$ Furthermore, the $\mathrm{Pb}$ signal is analyzed to rule out the presence of $\mathrm{MAPbI}_{3}$ in the tape during the delamination process. The typical value range for the $\mathrm{Pb}$ signal is marked in Figure $2 \mathrm{~d}$, but no signal was observed in this range; hence, the presence of $\mathrm{Pb}$ in the $\mathrm{Ag}$ contact is negligible, further confirming the formation of oxidized Ag in the form of AgI.

We then use the electrical technique IS to understand if it is possible to monitor the formation of $\mathrm{AgI}$ in terms of the interfacial resistance associated to this specific layer. For devices containing adequate extraction layers, the IS response in the dark shows two arcs (Figure 3a). A suitable equivalent circuit used to fit the spectra is shown in Figure $3 \mathrm{~b}$. The highfrequency (HF) arc is connected to the dielectric capacitance of the perovskite $\left(\mathrm{C}_{\mathrm{HF}}\right)$ and the resistance $\left(\mathrm{R}_{\mathrm{HF}}\right)$ containing contributions from both the perovskite and contacts. ${ }^{26} \mathrm{We}$ have previously observed that a modification in the $R_{H F}$ is a clear sign of a change in the doping concentration profile in the perovskite layer. ${ }^{36}$ Alternatively, the low-frequency (LF) arc is intimately connected to the resistance of the contacts $\left(\mathrm{R}_{\mathrm{LF}}\right)$ and the capacitance $\left(\mathrm{C}_{\mathrm{LF}}\right)$ arising from the accumulation of ions at the contacts in the form of double-layer capacitance. An observable noise and a modification of the LF resistance is a clear sign of reactivity between the migrating ions and the contacts.

Figure 3 shows the comparison between the IS spectra of the $\mathrm{Au}$ reference device and the device containing a buffer layer of Ag following the measurement sequence of $0 \mathrm{~V} \rightarrow 200 \mathrm{mV} \rightarrow$ $0 \mathrm{~V}$. The corresponding fitted resistances are tabulated in Table 1 . The reference device containing Au in direct contact

Table 1. Selected Fitted Resistance Values of Spectra Shown in Figure 3

\begin{tabular}{lccccc} 
& \multicolumn{2}{c}{ fresh } & & \multicolumn{2}{c}{ polarized } \\
\cline { 2 - 3 } \cline { 5 - 6 } & $\mathrm{R}_{\mathrm{HF}}(\mathrm{k} \Omega)$ & $\mathrm{R}_{\mathrm{LF}}(\mathrm{k} \Omega)$ & & $\mathrm{R}_{\mathrm{HF}}(\mathrm{k} \Omega)$ & $\mathrm{R}_{\mathrm{LF}}(\mathrm{k} \Omega)$ \\
$\mathrm{Au} \mathrm{ref}$ & 136 & 787 & & 67 & 36 \\
$\mathrm{Ag}(1$ st cycle $)$ & 2.9 & 4490 & & 2.9 & 437 \\
$\mathrm{Ag}\left(2^{\text {nd }}\right.$ cycle $)$ & 2.8 & 3580 & & 3.0 & 3640 \\
\hline
\end{tabular}

with Spiro-OMeTAD (Figure 1a) shows two arcs that reduce in resistance under an applied bias $\left(\mathrm{V}_{\mathrm{DC}}=200 \mathrm{mV}\right)$. However, comparing the initial and final responses at $0 \mathrm{~V}$, both the HF and $L F$ arcs show different resistance values. The $R_{H F}$ and $R_{L F}$ values reduce by a factor of 2 and 22, respectively. By the analysis of the HF arc, we can conclude that the doping density in the perovskite layer changes during the application of $\mathrm{V}_{\mathrm{DC}}=$ $200 \mathrm{mV}$. More importantly, the LF response, which is purely connected with the contacts, is not stable, leading to noise and highly distorted semicircles with a reduction in resistance for data points that take a long time to be recorded, see the inset 
in Figure 3a. All three observations support that the chemical reaction between migrating ions is incomplete and is taking place in the same time scale as the IS measurement. ${ }^{23,37}$ In contrast, the response of a device containing a buffer layer of $\mathrm{Ag}$ is markedly different. In particular, the HF arc does not change in magnitude during the application of a voltage bias, indicating that the perovskite does not change the doping concentration. We propose that the excess defects are either already immobilized by the chemical reaction with $\mathrm{Ag}$, as supported by XPS measurements of fresh devices (Supporting Information), or in transit in the Spiro-OMeTAD layer. In comparison to the reference device, the LF arc is stable, noise is not present, and arcs are not distorted, indicating that the chemical reaction with the remaining available ions is faster than the IS measurement. The LF arc reduces its resistance as observed for the reference device but, in this case, only by a factor of 10. The completion of the chemical reaction with ions, which could be in transit in the Spiro-OMeTAD layer, can be promoted by repeating the measurement sequence (following the sequence $0 \mathrm{~V} \rightarrow 200 \mathrm{mV} \rightarrow 0 \mathrm{~V}$ ) once again for the same device (Figure 3e,f). During this second round, the three measurements overlap perfectly in the HF and LF regions, indicating that the chemical reaction with the available ions has come to an end. We further highlight that the LF arc is related to slow cycling. Therefore, as we promote the chemical reaction, it appears that we promote the depletion of iodide ions close to the interface generating a favorable electronic situation (e.g., band bending) and this is the reason for the reduced hysteresis.

Selection Rules of Other Prospective Buffer Layer Materials and the Effect on the Perovskite Layer. We now explain the reasoning behind the selection of $\mathrm{Ag}$ as the buffer layer in order to provide the reader with a tool to select other prospective materials. We note that in order to discuss the electrochemistry, other tools such as theoretical calculations are better suited for this purpose but this is beyond our scope. In our work, we have used the electron work function of the metals as a first approximation to understand the reactivity of the buffer layer. In this respect, taking into account the work function difference between gold $(5.22 \mathrm{eV})$ and silver (4.36 $\mathrm{eV}$ ), with a difference of $\sim 0.8 \mathrm{~V}$, one can clearly infer that the $\mathrm{Ag}$ buffer electrode oxidizes more favorably than $\mathrm{Au}^{38}$ It is also important to recall that a redox pair is needed to complete the proposed semireaction. Due to the multilayer nature of the photovoltaic device, there are numerous candidates that can act as a redox pair in such a reaction, that is, Spiro-OMeTAD to Spiro-OMeTAD ${ }^{\bullet+}, 39,40 \mathrm{~Pb}^{2+}$ to $\mathrm{Pb},{ }^{19,41}$ or all species related to iodine chemistry $\left(\mathrm{I}_{2}\right.$ and $\left.\mathrm{I}_{3}{ }^{-}\right) \cdot{ }^{19,42}$ The task of detecting the type of species formed for the complementary reaction of AgI is extremely difficult and is beyond the scope of this work. Note that the internal photovoltage of the perovskite $(\sim 1.0 \mathrm{~V})$ is sufficiently high to promote these electrochemical reactions spontaneously. Therefore, Ag seems to be a suitable candidate to promote the irreversible oxidation in the presence of iodide ions.

In addition, the perovskite layer can be partially depleted of the migrating ions and can lead to the irreversible degradation of the perovskite material. This degradation due to the formation of AgI has been reported for thick Ag layers, wherein poor iodine-containing species such as $\mathrm{PbI}_{2}, \mathrm{PbO}$, or iodine vacancies $\left(\mathrm{V}_{\mathrm{I}}^{+}\right.$in the form of undercoordinated $\left.\mathrm{Pb}\right)$ would form within the perovskite layer. ${ }^{14}$ Therefore, in the selection of new materials, the reader also needs to take into account the thickness of the buffer layer. Thick buffer layers can lead to a large degree of iodine depletion in the perovskite material, which poses a negative impact on the device performance. In addition, the thick buffer layer can form considerably thick iodine-containing species (i.e., AgI in this work) leading to a higher series resistance in the device.

\section{CONCLUSIONS}

Overall, we have shown that the use of a thin layer of $\mathrm{Ag}$ between Spiro-OMeTAD and Au is very useful to passivate the contact/perovskite interface by the reaction of migrating ions that are close to the perovskite/Spiro-OMeTAD interface in the form of AgI. After the chemical reaction has been promoted, the low iodide ion activity at the interface generates a favorable electronic situation (e.g., band bending) and this is the reason for the reduced hysteresis. Therefore, hysteresis and enhanced stability of the device are promoted by the irreversible formation of AgI. Immobilization of ions is probed by XPS analysis and IS in working devices, revealing the kinetics of such chemical reactions. Further work using more stable perovskite formulations is in progress in our research group.

\section{ASSOCIATED CONTENT}

\section{Supporting Information}

The Supporting Information is available free of charge at https://pubs.acs.org/doi/10.1021/acsaem.0c01804.

Detailed experimental section, statistics of performance parameters, polarization protocol and IS measurement, degradation experiments, and XPS measurements (PDF)

\section{AUTHOR INFORMATION}

\section{Corresponding Authors}

Masoud Salavati-Niasari - Institute of Nano Science and Nano Technology, University of Kashan, Kashan, I. R. Iran; (i) orcid.org/0000-0002-7356-7249; Email: salavati@

kashanu.ac.ir

Antonio Guerrero - Institute of Advanced Materials, Universitat Jaume I, Castello 12006, Spain; 이이.org/ 0000-0001-8602-1248; Email: aguerrer@uji.es

\section{Authors}

Hakimeh Teymourinia - Institute of Advanced Materials, Universitat Jaume I, Castello 12006, Spain; Institute of Nano Science and Nano Technology, University of Kashan, Kashan, I. R. Iran

Cedric Gonzales - Institute of Advanced Materials, Universitat Jaume I, Castello 12006, Spain; ○ orcid.org/ 0000-0002-6550-2007

Juan Jesús Gallardo - Departamento de Química Física, Facultad de Ciencias, Universidad de Cádiz, Puerto Real (Cádiz) E-11510, Spain

Juan Bisquert - Institute of Advanced Materials, Universitat

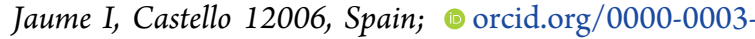
4987-4887

Javier Navas - Departamento de Química Física, Facultad de Ciencias, Universidad de Cádiz, Puerto Real (Cádiz) E11510, Spain; 이이이.org/0000-0001-7569-0809

Complete contact information is available at:

https://pubs.acs.org/10.1021/acsaem.0c01804 


\section{Author Contributions}

${ }^{\dagger}$ H.T. and C.G. contributed equally.

\section{Notes}

The authors declare no competing financial interest.

\section{ACKNOWLEDGMENTS}

We acknowledge financial support by Generalitat Valenciana for Prometeo (PROMETEO/2020/028) and Grisolia (GRISOLIAP/2019/048) grants from the Andalusian Government (SOL-201800107510-TRA). The council of Iran National Science Foundation (97017837) and the University of Kashan (159271/811290) are acknowledged for financial support.

\section{REFERENCES}

(1) NREL, B. R.-C. E., https://www.nrel.gov/pv/cell-efficiency.html (accessed: 31 October 2020).

(2) Leguy, A. M. A.; Hu, Y.; Campoy-Quiles, M.; Alonso, M. I.; Weber, O. J.; Azarhoosh, P.; van Schilfgaarde, M.; Weller, M. T.; Bein, T.; Nelson, J.; Docampo, P.; Barnes, P. R. F. Reversible Hydration of $\mathrm{CH}_{3} \mathrm{NH}_{3} \mathrm{PbI}_{3}$ in Films, Single Crystals, and Solar Cells. Chem. Mater. 2015, 27, 3397-3407.

(3) Motti, S. G.; Meggiolaro, D.; Barker, A. J.; Mosconi, E.; Perini, C. A. R.; Ball, J. M.; Gandini, M.; Kim, M.; De Angelis, F.; Petrozza, A. Controlling Competing Photochemical Reactions Stabilizes Perovskite Solar Cells. Nat. Photonics 2019, 13, 532.

(4) Turren-Cruz, S.-H.; Hagfeldt, A.; Saliba, M. Methylammoniumfree, High-performance and Stable Perovskite Solar Cells on a Planar Architecture. Science 2018, 362, 449.

(5) Wang, H.; Guerrero, A.; Bou, A.; Al-Mayouf, A. M.; Bisquert, J. Kinetic and Material Properties of Interfaces Governing Slow Response and Long Timescale Phenomena in Perovskite Solar Cells. Energy Environ. Sci. 2019, 12, 2054-2079.

(6) Domanski, K.; Roose, B.; Matsui, T.; Saliba, M.; Turren-Cruz, S.H.; Correa-Baena, J.-P.; Roldan Carmona, C.; Richardson, G.; Foster, J.; De Angelis, F.; Ball, J.; Petrozza, A.; Mine, N.; Nazeeruddin, M. K.; Tress, W.; Gratzel, M.; Steiner, U.; Hagfeldt, A.; Abate, A. Migration of Cations Induces Reversible Performance Losses over Day/night Cycling in Perovskite Solar Cells. Energy Environ. Sci. 2017, 10, 604613.

(7) Juarez-Perez, E. J.; Hawash, Z.; Raga, S. R.; Ono, L. K.; Qi, Y. Thermal Degradation of $\mathrm{CH}_{3} \mathrm{NH}_{3} \mathrm{PbI}_{3}$ Perovskite into $\mathrm{NH}_{3}$ and $\mathrm{CH}_{3} \mathrm{I}$ Gases Observed by Coupled Thermogravimetry-mass Spectrometry Analysis. Energy Environ. Sci. 2016, 9, 3406-3410.

(8) Bai, S.; Da, P.; Li, C.; Wang, Z.; Yuan, Z.; Fu, F.; Kawecki, M.; Liu, X.; Sakai, N.; Wang, J. T.-W.; Huettner, S.; Buecheler, S.; Fahlman, M.; Gao, F.; Snaith, H. J. Planar Perovskite Solar Cells with Long-term Stability using Ionic Liquid Additives. Nature 2019, 571, 245-250.

(9) Guerrero, A.; You, J.; Aranda, C.; Kang, Y. S.; Garcia-Belmonte, G.; Zhou, H.; Bisquert, J.; Yang, Y. Interfacial Degradation of Planar Lead Halide Perovskite Solar Cells. ACS Nano 2016, 10, 218-224.

(10) Sanehira, E. M.; Tremolet de Villers, B. J.; Schulz, P.; Reese, M. O.; Ferrere, S.; Zhu, K.; Lin, L. Y.; Berry, J. J.; Luther, J. M. Influence of Electrode Interfaces on the Stability of Perovskite Solar Cells: Reduced Degradation Using MoOx/Al for Hole Collection. ACS Energy Lett. 2016, 1, 38-45.

(11) Kim, G. Y.; Senocrate, A.; Yang, T.-Y.; Gregori, G.; Grätzel, M.; Maier, J. Large Tunable Photoeffect on Ion Conduction in Halide Perovskites and Implications for Photodecomposition. Nat. Mater. 2018, 17, 445-449.

(12) Senocrate, A.; Moudrakovski, I.; Kim, G. Y.; Yang, T.-Y.; Gregori, G.; Grätzel, M.; Maier, J. The Nature of Ion Conduction in Methylammonium Lead Iodide: A Multimethod Approach. Angew. Chem., Int. Ed. 2017, 56, 7755-7759.

(13) Thampy, S.; Zhang, B.; Hong, K.-H.; Cho, K.; Hsu, J. W. P. Altered Stability and Degradation Pathway of $\mathrm{CH}_{3} \mathrm{NH}_{3} \mathrm{PbI}_{3}$ in Contact with Metal Oxide. ACS Energy Lett. 2020, 5, 1147-1152.
(14) Kato, Y.; Ono, L. K.; Lee, M. V.; Wang, S.; Raga, S. R.; Qi, Y. Silver Iodide Formation in Methyl Ammonium Lead Iodide Perovskite Solar Cells with Silver Top Electrodes. Adv. Mater. Interfaces 2015, 2, No. 1500195.

(15) Besleaga, C.; Abramiuc, L. E.; Stancu, V.; Tomulescu, A. G.; Sima, M.; Trinca, L.; Plugaru, N.; Pintilie, L.; Nemnes, G. A.; Iliescu, M.; Svavarsson, H. G.; Manolescu, A.; Pintilie, I. Iodine Migration and Degradation of Perovskite Solar Cells Enhanced by Metallic Electrodes. J. Phys. Chem. Lett. 2016, 7, 5168-5175.

(16) Pospisil, J.; Guerrero, A.; Zmeskal, O.; Weiter, M.; Gallardo, J. J.; Navas, J.; Garcia-Belmonte, G. Reversible Formation of Gold Halides in Single-Crystal Hybrid-Perovskite/Au Interface upon Biasing and Effect on Electronic Carrier Injection. Adv. Funct. Mater. 2019, 29, No. 1900881.

(17) Carrillo, J.; Guerrero, A.; Rahimnejad, S.; Almora, O.; Zarazua, I.; Mas-Marza, E.; Bisquert, J.; Garcia-Belmonte, G. Ionic Reactivity at Contacts and Aging of Methylammonium Lead Triiodide Perovskite Solar Cell. Adv. Energy Mater. 2016, 6, No. 1502246.

(18) Yang, T.-Y.; Jeon, N. J.; Shin, H.-W.; Shin, S. S.; Kim, Y. Y.; Seo, J. Achieving Long-Term Operational Stability of Perovskite Solar Cells with a Stabilized Efficiency Exceeding 20\% after 1000 h. Adv. Sci. 2019, 6, No. 1900528.

(19) Gunasekaran, R. K.; Chinnadurai, D.; Selvaraj, A. R.; Rajendiran, R.; Senthil, K.; Prabakar, K. Revealing the SelfDegradation Mechanisms in Methylammonium Lead Iodide Perovskites in Dark and Vacuum. ChemPhysChem 2018, 19, 1507-1513.

(20) Zhao, Y.; Zhou, W.; Tan, H.; Fu, R.; Li, Q.; Lin, F.; Yu, D.; Walters, G.; Sargent, E. H.; Zhao, Q. Mobile-Ion-Induced Degradation of Organic Hole-Selective Layers in Perovskite Solar Cells. J. Phys. Chem. C 2017, 121, 14517-14523.

(21) Cacovich, S.; Ciná, L.; Matteocci, F.; Divitini, G.; Midgley, P. A.; Di Carlo, A.; Ducati, C. Gold and Iodine Diffusion in Large Area Perovskite Solar Cells under Illumination. Nanoscale 2017, 9, 47004706.

(22) Svanström, S.; Jacobsson, T. J.; Boschloo, G.; Johansson, E. M. J.; Rensmo, H.; Cappel, U. B. Degradation Mechanism of Silver Metal Deposited on Lead Halide Perovskites. ACS Appl. Mater. Interfaces 2020, 12, 7212-7221.

(23) Solanki, A.; Guerrero, A.; Zhang, Q.; Bisquert, J.; Sum, T. C. Interfacial Mechanism for Efficient Resistive Switching in Ruddlesden-Popper Perovskites for Non-volatile Memories. J. Phys. Chem. Lett. 2020, 11, 463-470.

(24) Peng, W.; Aranda, C.; Bakr, O. M.; Garcia-Belmonte, G.; Bisquert, J.; Guerrero, A. Quantification of Ionic Diffusion in Lead Halide Perovskite Single Crystals. ACS Energy Lett. 2018, 3, 14771481.

(25) Correa-Baena, J.-P.; Turren-Cruz, S.-H.; Tress, W.; Hagfeldt, A.; Aranda, C.; Shooshtari, L.; Bisquert, J.; Guerrero, A. Changes from Bulk to Surface Recombination Mechanisms between Pristine and Cycled Perovskite Solar Cells. ACS Energy Lett. 2017, 2, 681-688.

(26) Guerrero, A.; Garcia-Belmonte, G.; Mora-Sero, I.; Bisquert, J.; Kang, Y. S.; Jacobsson, T. J.; Correa-Baena, J.-P.; Hagfeldt, A. Properties of Contact and Bulk Impedances in Hybrid Lead Halide Perovskite Solar Cells Including Inductive Loop Elements. J. Phys. Chem. C 2016, 120, 8023-8032.

(27) Guerrero, A.; Bou, A.; Matt, G.; Almora, O.; Heumüller, T.; Garcia-Belmonte, G.; Bisquert, J.; Hou, Y.; Brabec, C. Switching Off Hysteresis in Perovskite Solar Cells by Fine-Tuning Energy Levels of Extraction Layers. Adv. Energy Mater. 2018, 8, No. 1703376.

(28) Tress, W.; Marinova, N.; Moehl, T.; Zakeeruddin, S. M.; Nazeeruddin, M. K.; Gratzel, M. Understanding the Rate-dependent J-V Hysteresis, Slow Time Component, and Aging in $\mathrm{CH}_{3} \mathrm{NH}_{3} \mathrm{PbI}_{3}$ Perovskite Solar Cells: The Role of a Compensated Electric Field. Energy Environ. Sci. 2015, 8, 995-1004.

(29) Ravishankar, S.; Almora, O.; Echeverría-Arrondo, C.; Ghahremanirad, E.; Aranda, C.; Guerrero, A.; Fabregat-Santiago, F.; Zaban, A.; Garcia-Belmonte, G.; Bisquert, J. Surface Polarization Model for the Dynamic Hysteresis of Perovskite Solar Cells. J. Phys. Chem. Lett. 2017, 8, 915-921. 
(30) Naumkin, A. V.; Kraut-Vass, A.; Gaarenstroom, S. W.; Powell, C. J., in NIST Standard Reference Database 20, Version 4.1, Gaithersburg 2012.

(31) Eckardt, H.; Fritsche, L. Theoretical Explanation of the Xps Satellite Structure of Elementary Metals - Application to Ag. Solid State Commun. 1985, 54, 405-407.

(32) Zheng, Z.; Liu, A. R.; Wang, S. M.; Huang, B. J.; Ma, X. M.; Zhao, H.; Li, D. P.; Zhang, L. Z. In situ Fabrication of AgI Films on Various Substrates. Mater. Res. Bull. 2008, 43, 2491-2496.

(33) Jonjana, S.; Phuruangrat, A.; Thongtem, S.; Thongtem, T. Synthesis of $\mathrm{AgI} / \mathrm{Bi}_{2} \mathrm{MoO}_{6}$ Heterojunctions and Their Photoactivity Enhancement Driven by Visible Light. Mater. Lett. 2016, 175, 75-78.

(34) Ye, H. F.; Lin, H. L.; Cao, J.; Chen, S. F.; Chen, Y. Enhanced Visible Light Photocatalytic Activity and Mechanism of $\mathrm{BiPO}_{4}$ Nanorods Modified with AgI Nanoparticles. J. Mol. Catal. A: Chem. 2015, 397, 85-92.

(35) Moulder, J. F.; Stickle, W. F.; Sobol, P. E.; Bomben, K. D., Handbook of X-ray Photoelectron Spectroscopy. Perkin-Elmer Corporation, Physical Electronics Division: Eden Prairie, Minnesota, USA, 1992.

(36) Li, C.; Guerrero, A.; Huettner, S.; Bisquert, J. Unravelling the Role of Vacancies in Lead Halide Perovskite through Electrical Switching of Photoluminescence. Nat. Commun. 2018, 9, 5113.

(37) Aranda, C.; Bisquert, J.; Guerrero, A. Impedance Spectroscopy of Perovskite/contact Interface: Beneficial Chemical Reactivity Effect. J. Chem. Phys. 2019, 151, 124201.

(38) Derry, G. N.; Kern, M. E.; Worth, E. H. Recommended Values of Clean Metal Surface Work Functions. J. Vac. Sci. Technol., A 2015, 33, No. 060801.

(39) Sanchez, R. S.; Mas-Marza, E. Light-induced Effects on SpiroOMeTAD Films and Hybrid Lead Halide Perovskite Solar Cells. Sol. Energy Mater. Sol. Cells 2016, 158, 189.

(40) Wang, H.; Xu, M.; Liu, G.; Li, X.; Xiang, P.; Ku, Z.; Rong, Y.; Liu, L.; Hu, M.; Yang, Y.; Han, H. Effect of Photo-doping on Performance for Solid-state Dye-sensitized Solar Cell Based on 2,2'7,7'-tetrakis-(N,N-di-p-methoxyphenyl-amine)-9,9'-spirobifluorene and Carbon Counter Electrode. Electrochim. Acta 2013, 99, 238.

(41) Raga, S. R.; Jung, M.-C.; Lee, M. V.; Leyden, M. R.; Kato, Y.; Qi, Y. Influence of Air Annealing on High Efficiency Planar Structure Perovskite Solar Cells. Chem. Mater. 2015, 27, 1597.

(42) Meggiolaro, D.; Motti, S. G.; Mosconi, E.; Barker, A. J.; Ball, J.; Andrea Riccardo Perini, C.; Deschler, F.; Petrozza, A.; De Angelis, F. Iodine Chemistry Determines the Defect Tolerance of Lead-halide Perovskites. Energy Environ. Sci. 2018, 11, 702. 